\title{
A REMARK ON PARTIAL SUMS INVOLVING THE MÖBIUS FUNCTION
}

\author{
TERENCE TAO
}

(Received 29 August 2009)

\begin{abstract}
Let $\langle\mathcal{P}\rangle \subset \mathbf{N}$ be a multiplicative subsemigroup of the natural numbers $\mathbf{N}=\{1,2,3, \ldots\}$ generated by an arbitrary set $\mathcal{P}$ of primes (finite or infinite). We give an elementary proof that the partial sums $\sum_{n \in\langle\mathcal{P}\rangle: n \leq x}(\mu(n)) / n$ are bounded in magnitude by 1 . With the aid of the prime number theorem, we also show that these sums converge to $\prod_{p \in \mathcal{P}}(1-(1 / p))$ (the case where $\mathcal{P}$ is all the primes is a wellknown observation of Landau). Interestingly, this convergence holds even in the presence of nontrivial zeros and poles of the associated zeta function $\zeta \mathcal{P}(s):=\prod_{p \in \mathcal{P}}\left(1-\left(1 / p^{s}\right)\right)^{-1}$ on the line $\{\operatorname{Re}(s)=1\}$. As equivalent forms of the first inequality, we have $\left|\sum_{n \leq x}:(n, P)=1(\mu(n)) / n\right| \leq 1,\left|\sum_{n \mid N: n \leq x}(\mu(n)) / n\right| \leq 1$, and $\left|\sum_{n \leq x}(\mu(m n)) / n\right| \leq 1$ for all $m, x, N, P \geq 1$.
\end{abstract}

2000 Mathematics subject classification: primary 11A25.

Keywords and phrases: Möbius function, prime number theorem, effective inequalities.

\section{Introduction}

Let $\mathbf{N}:=\{1,2, \ldots\}$ be the natural numbers, and let $\mu: \mathbf{N} \rightarrow\{-1,0,+1\}$ be the Möbius function, thus $\mu(n)=(-1)^{k}$ when $n$ is the product of $k$ distinct primes, and $\mu(n)=0$ otherwise. Landau [4] made the elementary observation that the prime number theorem ${ }^{1}$

$$
\sum_{p \leq x} 1=(1+o(1)) \frac{x}{\log x}
$$

is equivalent to the conditional convergence of the infinite sum $\sum_{n}((\mu(n)) / n)=0$, thus

$$
\sum_{n \leq x} \frac{\mu(n)}{n}=o(1) .
$$

The author is supported by NSF Research Award DMS-0649473, the NSF Waterman award and a grant from the MacArthur Foundation.

${ }^{1}$ We adopt the convention that $n$ always ranges over the natural numbers, and $p$ over prime numbers, unless otherwise stated. The notation $o(1)$ denotes any quantity that converges to zero as $x \rightarrow \infty$, holding all other parameters fixed.

(C) 2010 Australian Mathematical Publishing Association Inc. 0004-9727/2010 \$16.00 
As is well known, (1.1) and the prime number theorem are also both equivalent to the fact that the Riemann zeta function

$$
\zeta(s):=\sum_{n} \frac{1}{n^{s}}=\prod_{p}\left(1-\frac{1}{p^{s}}\right)^{-1}
$$

has a simple pole at $s=1$ and no zeros or poles elsewhere on the line $\{\operatorname{Re}(s)=1\}$. See [1] for further discussion of this and other equivalences.

On the other hand, there is the elementary bound

$$
\left|\sum_{n \leq x} \frac{\mu(n)}{n}\right| \leq 1
$$

for all $x$. Indeed, to see this we may assume without loss of generality that $x$ is a natural number, and then sum the Möbius inversion formula ${ }^{1} 1_{n=1}=\sum_{d \mid n} \mu(d)$ from 1 to $x$ to obtain the identity

$$
1=\sum_{d \leq x} \mu(d)\left\lfloor\frac{x}{d}\right\rfloor=\sum_{d \leq x} \mu(d) \frac{x}{d}-\sum_{d<x} \mu(d)\left\{\frac{x}{d}\right\}
$$

where $\{y\}:=y-\lfloor y\rfloor$ is the fractional part of $y$. Using the trivial bound $|\mu(d)\{x / d\}|$ $\leq 1$ and the triangle inequality, one obtains (1.3). The bound (1.3) is of course attained with equality when $x=1$.

In this paper we investigate the analogue of these facts when we 'turn off' some of the primes in $\mathbf{N}$. More precisely, we consider an arbitrary set $\mathcal{P}$ of primes (either finite or infinite), and let $\langle\mathcal{P}\rangle \subset \mathbf{N}$ be the multiplicative semigroup generated by $\mathcal{P}$ (that is, the set of natural numbers whose prime factors all lie in $\mathcal{P}$ ). We will study the behaviour of the sum

$$
\sum_{n \in\langle\mathcal{P}\rangle: n \leq x} \frac{\mu(n)}{n}
$$

in $x$ and $\mathcal{P}$. The analogue of the Riemann zeta function (1.2) is then the Burgess zeta function $\zeta_{\mathcal{P}}$, defined for $\operatorname{Re}(s)>1$ by the formula

$$
\zeta \mathcal{P}(s):=\sum_{n \in\langle\mathcal{P}\rangle} \frac{1}{n^{s}}=\prod_{p \in \mathcal{P}}\left(1-\frac{1}{p^{s}}\right)^{-1} .
$$

Note that as $\mathcal{P}$ is arbitrary, there need not be any asymptotic formula for the prime counting function $\sum_{p \in \mathcal{P}, p<x} 1$. For similar reasons, there need not be any meromorphic continuation of $\zeta_{\mathcal{P}}$ beyond the region $\{\operatorname{Re}(s)>1\}$; for instance, one can easily construct a set $\mathcal{P}$ for which $\zeta_{\mathcal{P}}(s)$ blows up as $s \rightarrow 1^{+}$at an intermediate rate between 1 and $1 /(s-1)$, which is not consistent with any meromorphic continuation at $s=1$.

\footnotetext{
$\overline{{ }^{1} \text { We write } 1_{E}}$ to denote the indicator of a statement $E$, thus $1_{E}=1$ when $E$ is true and $1_{E}=0$ otherwise.
} 
Related to this, the zeta function $\zeta_{\mathcal{P}}(s)$ can develop zeros or singularities on the line $\operatorname{Re}(s)=1$. Indeed, observe for $\operatorname{Re}(s)>1$ that

$$
\log \left|\zeta_{\mathcal{P}}(s)\right|=-\sum_{p \in \mathcal{P}} \log \left|1-\frac{1}{p^{s}}\right|=\sum_{p \in \mathcal{P}} \frac{1}{p^{s}}+O(1) .
$$

For any nonzero real number $t$, if one sets $\mathcal{P}$ to be those primes $p$ for which $\{(t \log p) / 2 \pi\} \leq 0.1$ (say), then one can easily check that $\left|\zeta_{\mathcal{P}}(1+i t+\varepsilon)\right| \rightarrow \infty$ as $\varepsilon \rightarrow 0$; similarly, setting $\mathcal{P}$ instead to be those primes for which $\{((t \log p) / 2 \pi)-$ $1 / 2\} \leq 0.1$, then $\left|\zeta_{\mathcal{P}}(1+i t+\varepsilon)\right| \rightarrow 0$. A modification of these examples shows that $\zeta_{\mathcal{P}}$ need not have a meromorphic continuation at $1+i t$ for a fixed $t$, and with a bit more effort one can concoct a $\mathcal{P}$ for which $\zeta_{\mathcal{P}}$ has no continuation at $1+i t$ for any $t$; we omit the details.

Despite this, one can $^{1}$ generalize the statements (1.1), (1.3) to arbitrary $\mathcal{P}$. We first prove the generalization of (1.3), which is surprisingly elementary.

THEOREM 1.1 (Elementary bound). For any $\mathcal{P}$ and $x$,

$$
\left|\sum_{n \in\langle\mathcal{P}\rangle: n \leq x} \frac{\mu(n)}{n}\right| \leq 1
$$

PROOF. We may assume of course that $x$ is a natural number. We may also assume that

$$
\sum_{n \in\langle\mathcal{P}\rangle: n \leq x} \frac{1}{n}>1
$$

since the claim is immediate from the triangle inequality otherwise.

Let $\mathcal{P}^{\prime}$ be the set of primes not in $\mathcal{P}$. From Möbius inversion, for all natural numbers $n$,

$$
1_{n \in\left\langle\mathcal{P}^{\prime}\right\rangle}=\sum_{d \in\langle\mathcal{P}\rangle: d \mid n} \mu(d)
$$

summing this over all $n \leq x$ as in the proof of (1.3) yields

$$
\sum_{n \in\left\langle\mathcal{P}^{\prime}\right\rangle: n \leq x} 1=\sum_{d \in\langle\mathcal{P}\rangle: d \leq x} \mu(d) \frac{x}{d}-\sum_{d \in\langle\mathcal{P}\rangle: d \leq x} \mu(d)\left\{\frac{x}{d}\right\} .
$$

Using the bound

$$
\left|\mu(d)\left\{\frac{x}{d}\right\}\right| \leq 1-\frac{1}{d}
$$

we conclude that

$$
\left|x \sum_{n \in\langle\mathcal{P}\rangle: n \leq x} \frac{\mu(n)}{n}\right| \leq \sum_{n \in\left\langle\mathcal{P}^{\prime}\right\rangle: n \leq x} 1+\sum_{n \in\langle\mathcal{P}\rangle: n \leq x} 1-\sum_{n \in\langle\mathcal{P}\rangle: n \leq x} \frac{1}{n} .
$$

Since $\langle\mathcal{P}\rangle$ and $\left\langle\mathcal{P}^{\prime}\right\rangle$ overlap only at 1 , the claim now follows from (1.7).

\footnotetext{
${ }^{1}$ Note added in proof: as pointed out to us after the submission of this paper, these generalizations were essentially contained in [2] and [5], respectively; see Remarks 1.2 and 1.5. We hope, however, that this paper continues to play an expository role in highlighting these elementary results.
} 
REMARK 1.2. A bound very similar to (1.6) was also observed in [2]. In particular, the refinement

$$
x \sum_{n \in\langle\mathcal{P}\rangle: n \leq x} \frac{\mu(n)}{n}=\sum_{n \in\left\langle\mathcal{P}^{\prime}\right\rangle: n \leq x} 1+(1-\gamma) \sum_{n \in\langle\mathcal{P}\rangle: n \leq x} \mu(n)+O\left(\frac{x}{\log ^{1 / 5} x}\right)
$$

to (1.8) was obtained as a special case of [2, Theorem 3.1]. The lower bound of -1 for $\sum_{n \in\langle\mathcal{P}\rangle: n \leq x}(\mu(n)) / n$ was also improved in [2, Theorem 2] to

$$
1-2 \log (1+\sqrt{e})+4 \int_{1}^{\sqrt{e}} \frac{\log t}{t+1} d t+o(1)=-0.4553 \cdots+o(1),
$$

which is optimal except for the $o(1)$ term, with a characterization of those primes $\mathcal{P}$ for which the lower bound is attained.

As corollaries of Theorem 1.1 we have

$$
\left|\sum_{n \leq x:(n, P)=1} \frac{\mu(n)}{n}\right| \leq 1
$$

and

$$
\left|\sum_{n \mid N: n \leq x} \frac{\mu(n)}{n}\right| \leq 1
$$

for any $P, x, N \geq 1$; also, from the identity $\mu(m n)=\mu(m) \mu(n) 1_{(m, n)=1}$ one has

$$
\left|\sum_{n \leq x} \frac{\mu(m n)}{n}\right| \leq 1
$$

for any $m, x \geq 1$. These inequalities, which save a factor of $O(\log x)$ over the trivial bound, may be of some value in obtaining effective estimates in sieve theory or in exponential sums over the primes.

We now turn to the generalization of (1.1).

THEOREM 1.3 (Landau's theorem for arbitrary sets of primes). Let $\mathcal{P}$ be a set of primes. Then the sum $\sum_{n \in\langle\mathcal{P}\rangle}(\mu(n)) / n$ converges conditionally to $\prod_{p \in \mathcal{P}}(1-(1 / p))$, thus

$$
\sum_{n \in\langle\mathcal{P}\rangle: n \leq x} \frac{\mu(n)}{n}=\prod_{p \in \mathcal{P}}\left(1-\frac{1}{p}\right)+o(1)
$$

for all $x>0$, where the decay rate of the error $o(1)$ depends on $\mathcal{P}$. In particular, $\sum_{n \in\langle P\rangle}(\mu(n)) / n$ is conditionally convergent to zero if and only if $\sum_{p \in \mathcal{P}} 1 / p$ is infinite.

The proof of Theorem 1.3 is also elementary (except for its use of (1.1), which is of course the special case of (1.12) when $\mathcal{P}$ consists of all the primes). Interestingly, it is surprisingly difficult to replicate this elementary proof by zeta function methods, in large part due to the lack of meromorphic continuation alluded to earlier. 
REMARK 1.4. A classical result of Wirsing (see, for example, [3]) on mean values of multiplicative functions implies in particular that

$$
\frac{1}{x} \sum_{n \in\langle\mathcal{P}\rangle: n \leq x} \mu(n)=o(1) .
$$

This fact is also deducible from (1.12).

REMARK 1.5. Theorem 1.3 was also proven in [5] by a similar method; in fact, the result in [5] extends to arbitrary collections of prime ideals in algebraic number fields.

We remark that the decay rate $o(1)$ in (1.12) is not uniform in $\mathcal{P}$. For instance, if one takes $\mathcal{P}$ to be all the primes $p$ between $\sqrt{x}$ and $x$, one sees from Mertens' theorems that

$$
\sum_{n \in\langle\mathcal{P}\rangle: n \leq x} \frac{\mu(n)}{n}=1-\sum_{\sqrt{x} \leq p \leq x} \frac{1}{p}=1-\log 2+o(1)
$$

and

$$
\prod_{p \in \mathcal{P}}\left(1-\frac{1}{p}\right)=\frac{1}{2}+o(1)
$$

and so one can keep the error term $o(1)$ in (1.12) bounded away from zero even for arbitrarily large $x$, by choosing $\mathcal{P}$ depending on $x$. More precise statements of this nature can be found in [2].

Note that the above results do not hold when $\langle\mathcal{P}\rangle$ is replaced by a more general subsemigroup $G$ of the natural numbers (in which the generators are not necessarily prime). For instance ${ }^{1}$, if $G$ is the semigroup generated by the semiprimes (the products of two primes), then $\mu$ is either 0 or 1 and it is not difficult to see that $\sum_{n \in G}(\mu(n)) / n$ diverges. One reason for the bad behaviour of these sums is that the zeta function $\sum_{n \in G} 1 / n^{s}$ no longer has an Euler product.

It is also essential that $\mathcal{P}$ consist of natural numbers, rather than merely real numbers larger than 1 (as is the case in Beurling prime models), as the equal spacing of the integers is used in an essential way. For instance ${ }^{2}$, the inequality (1.6) fails when $\mathcal{P}=\{1.1,1.2,1.3\}$ and $x=1.3$.

\section{Proof of main theorem}

We now establish Theorem 1.3. Fix $\mathcal{P}$; we allow all implied constants in the asymptotic notation to depend on $\mathcal{P}$.

If $\sum_{p \in \mathcal{P}} 1 / p$ is finite, then from the monotone convergence theorem we see that

$$
\sum_{n \in\langle\mathcal{P}\rangle} \frac{1}{n}=\prod_{p \in \mathcal{P}}\left(1+\frac{1}{p-1}\right)
$$

\footnotetext{
${ }^{1}$ We thank Andrew Sutherland for this example.

${ }^{2}$ We thank Harold Diamond for this example.
} 
is absolutely convergent, and thus (by dominated convergence)

$$
\sum_{n \in\langle\mathcal{P}\rangle} \frac{\mu(n)}{n}=\prod_{p \in \mathcal{P}}\left(1-\frac{1}{p}\right)
$$

is conditionally convergent, giving the claim. Thus we shall assume that $\sum_{p \in \mathcal{P}} 1 / p$ is infinite, in which case our task is to show that

$$
\sum_{n \in\langle\mathcal{P}\rangle: n \leq x} \frac{\mu(n)}{n}=o(1)
$$

Let $\mathcal{P}^{\prime}:=\{p: p \notin \mathcal{P}\}$ be the complement of $\mathcal{P}$ in the primes. Suppose that $\sum_{p \in \mathcal{P}^{\prime}} 1 / p$ is also infinite, thus

$$
\prod_{p \in \mathcal{P}}\left(1-\frac{1}{p}\right)=\prod_{p \in \mathcal{P}^{\prime}}\left(1-\frac{1}{p}\right)=0 .
$$

From elementary sieve theory (the Legendre sieve), this implies that both $\langle\mathcal{P}\rangle$ and $\left\langle\mathcal{P}^{\prime}\right\rangle$ have asymptotic density zero, thus the right-hand side of (1.8) is $o(x)$, and (2.1) follows.

The last remaining case is when $\sum_{p \in \mathcal{P}^{\prime}} 1 / p$ is finite. In this case, we use Möbius inversion to write

$$
1_{n \in\langle\mathcal{P}\rangle}=\sum_{d \in\left\langle\mathcal{P}^{\prime}\right\rangle: d \mid n} \mu(d)
$$

and thus

$$
\sum_{n \in\langle\mathcal{P}\rangle: n \leq x} \frac{\mu(n)}{n}=\sum_{d \in\left\langle\mathcal{P}^{\prime}\right\rangle: d \leq x} \frac{\mu(d)}{d} \sum_{m \leq x / d} \frac{\mu(d m)}{m} .
$$

By (1.11),

$$
\frac{\mu(d)}{d} \sum_{m \leq x / d} \frac{\mu(d m)}{m}
$$

is bounded in magnitude by $1 / d$. As $\sum_{p \in \mathcal{P}^{\prime}} 1 / p$ is finite, $\sum_{d \in\left\langle\mathcal{P}^{\prime}\right\rangle} 1 / d$ is absolutely convergent, so by dominated convergence it suffices to show that

$$
\sum_{m \leq x} \frac{\mu(d m)}{m}=o(1)
$$

for each fixed $d$.

Fix $d$. If we take $\mathcal{P}_{d}$ to be the primes dividing $d$, we observe the Möbius inversion identity

$$
\begin{aligned}
\mu(d m) & =\mu(d) \mu(m) 1_{(d, m)=1} \\
& =\sum_{n \in\left\langle\mathcal{P}_{d}\right\rangle: n \mid m} \mu(d) \mu(m / n)
\end{aligned}
$$


and so

$$
\sum_{m \leq x} \frac{\mu(d m)}{m}=\mu(d) \sum_{n \in\left\langle\mathcal{P}_{d}\right\rangle: n \leq x} \frac{1}{n} \sum_{l \leq x / n} \frac{\mu(l)}{l} .
$$

Since $\sum_{n \in\left\langle\mathcal{P}_{d}\right\rangle} 1 / n$ is absolutely convergent, the claim now follows from (1.3), (1.1) and the dominated convergence theorem.

REMARK 2.1. By a convexity argument, one sees from (1.6) that

$$
\left|\sum_{n \leq x} \frac{\mu(n) a(n)}{n}\right| \leq 1
$$

for every multiplicative function $a: \mathbf{N} \rightarrow[0,1]$ taking values between zero and one (note that the expression in absolute values is affine-linear in $a(p)$ for each prime $p$, and (1.6) is the special case where the $a(p)$ take the extreme values of 0 and 1); see also [2]. It is also not difficult to adapt the arguments in this section to show that $\sum_{n=1}^{\infty}(\mu(n) a(n)) / n$ converges conditionally to $\prod_{p}(1-((a(p)) / p))$; we leave the details to the interested reader.

\section{Acknowledgements}

We thank Melvyn Nathanson and Keith Conrad for corrections, and Władysław Narkiewicz, Mariusz Skałba, Kannan Soundararajan for references. We are also indebted to the anonymous referee for corrections and suggestions.

\section{References}

[1] H. Diamond, 'Elementary methods in the study of the distribution of prime numbers', Bull. Amer. Math. Soc. 7 (1982), 553-589.

[2] A. Granville and K. Soundararajan, 'Negative values of truncations to $L(1, \chi)$ ', in: Analytic Number Theory: A Tribute to Gauss and Dirichlet, Clay Mathematics Proceedings, 7 (American Mathematical Society, Providence, RI, 2007).

[3] A. Hildebrand, 'On Wirsing's mean value theorem for multiplicative functions', Bull. London Math. Soc. 18 (1986), 147-152.

[4] E. Landau, 'Über den Zusammenhang einiger neuer Sätze der analytischen Zahlentheorie', Wiener Sitzungsberichte, Math. Klasse 115 (1906), 589-632.

[5] M. Skałba, 'On Euler-von Mangoldt's equation', Colloq. Math. 69 (1995), 143-145.

TERENCE TAO, Department of Mathematics, UCLA, Los Angeles CA 90095-1555, USA

e-mail: tao@math.ucla.edu 